\title{
Development of Stimuli-Responsive Multi-Block Amphiphiles
}

\author{
Takahiro Muraoka,* Shunichi Kawasaki, and Kazushi Kinbara* \\ Institute of Multidisciplinary Research for Advanced Materials, Tohoku University, \\ 2-1-1, Katahira, Aoba-ku, Sendai 980-8577, Japan \\ *E-mail: kinbara@tagen.tohoku.ac.jp
}

Keywords: amphiphile, extraction, protein, self-assembly

\section{Introduction}

Temperature-responsive compounds are useful to develop smart materials. Poly $(N$-isopropylacrylamide) (PNIPAM) is a representative temperature-responsive polymer that shows aggregation in an aqueous condition by increasing the hydrophobicity upon temperature elevation. ${ }^{1}$ Among various applications of this temperature-response, ${ }^{2}$ extraction is important for water purification to remove toxic substances, ${ }^{3}$ condensation of small organic molecules for analyses $^{4}$ and extraction of metal ions. ${ }^{5}$ Recently, we reported a temperature-responsive small amphiphile 1,1'-((2,5,8,11,14-pentaoxapentadecane1,15-diyl)-bis(2-phenyl-1,3-dioxane-5,5-diyl))bis(2, 5,8,11-tetraoxatridecan-13-ol) (1) that shows micrometer-scale aggregation in water upon heating due to a conformational change at the ethylene oxide units. ${ }^{6}$ The thermo-driven aggregation of 1 was useful for selective peptide extraction. In this study, we investigated protein extraction with $\mathbf{1}$ in comparison with PNIPAM.

\section{Methods}

\subsection{General}

${ }^{1} \mathrm{H}$ and ${ }^{13} \mathrm{C}$ nuclear magnetic resonance (NMR) spectra were recorded on $400 \mathrm{MHz}$ FT NMR Bruker BioSpin AVANCE III 400 spectrometer, 500 MHz FT NMR Bruker BioSpin AVANCE III 500, or $600 \mathrm{MHz}$ FT NMR Burker BioSpin AVANCEIII 600 , where the chemical shifts were determined with respect to tetramethylsilane (TMS), hexamethyldisilane (HMDS) or a residual non-deuterated solvent as an internal standard.

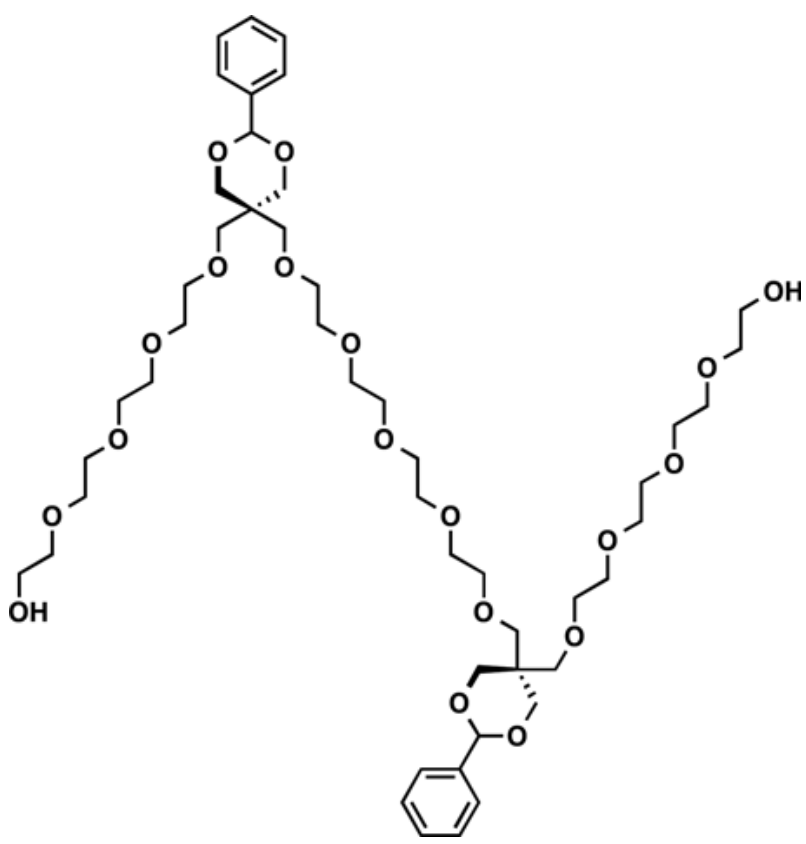

Fig. 1 Molecular structure of $1,1^{\prime}-((2,5,8,11,14-$ pentaoxapentadecane-1,15-diyl)bis(2-phenyl-1,3-dioxane -5,5-diyl))bis(2,5,8,11-tetraoxatridecan-13-ol) (1).

Matrix-assisted laser desorption/ionization time-of- flight mass (MALDI-TOF MS) spectrometry was performed in a reflector positive mode with $\alpha$-cyano-4-hydrocinnamic acid (CHCA) as a matrix on Bruker autoflex speed, and high resolution electrospray ionization (HR ESI) TOF 
MS spectra were recorded on Bruker micrOTOF-Q II-S1. Vortex was performed with Scientific Industries Vortex-Genie2. Absorption spectra were recorded on JASCO V-530 UV-Vis spectrophotometer with $1.0-\mathrm{mm}$ thick quartz quvette. Centrifugation was performed using TOMY MX-301 High Speed Refrigerated Micro Centrifuge under a regulated temperature. Temperature control for the protein extraction was performed with TAITEC aluminum block bath dry thermo unit DTU-1B.

\subsection{Reagents}

Deuterated solvents were purchased from Acros Organics. CHCA was purchased from Sigma-Aldrich. $\quad \operatorname{Poly}(N$-isopropylacrylamide) (PNIPAM) was purchased from POLYSIENCES (average molecular weight: 40000). Protein calibration standard I was purchased from Bruker Daltonics. Cytochrome C was purchased from Sigma-Aldrich. Deionized water (filtered through a $0.22 \mu \mathrm{m}$ membrane filter, $>18.2 \mathrm{M} \Omega \mathrm{cm}$ ) was purified in a Milli-Q system of Millipore.

\subsection{Synthesis}

1 was synthesized by oxidative deprotection of 4-methoxyphenyl groups of 1,15-bis(5-(15-(4methoxyphenyl)-2,5,8,11,14-pentaoxapentadecyl)2-phenyl-1,3-dioxan-5-yl)-2,5,8,11,14-pentaoxapentadecane with 2,3-dichloro-5,6-dicyano-1,4benzoquinone (DDQ) in dry $\mathrm{CH}_{2} \mathrm{Cl}_{2}$ at $0{ }^{\circ} \mathrm{C}$, which was unambiguously characterized by NMR and HR ESI MS analyses. The detailed experimental procedure has been reported in our previous paper. ${ }^{6}$

\subsection{Protein extraction}

The whole content of a tube of Protein calibration standard I was dissolved in $125-\mu \mathrm{L}$ water. An aliquot of $50 \mu \mathrm{L}$ was transferred into a 1.5-mL Eppendorf centrifuge tube, to which was added $1(21 \mu \mathrm{mol})$ or PNIPAM $(20.1 \mathrm{mg})$. The mixture was subjected to vortex twice for $10 \mathrm{~s}$ each at $20^{\circ} \mathrm{C}$ to afford a homogeneous mixture, which was then kept standing for $30 \mathrm{~min}$ at $40{ }^{\circ} \mathrm{C}$. The resulting turbid suspension was centrifuged for $10 \mathrm{~s}$
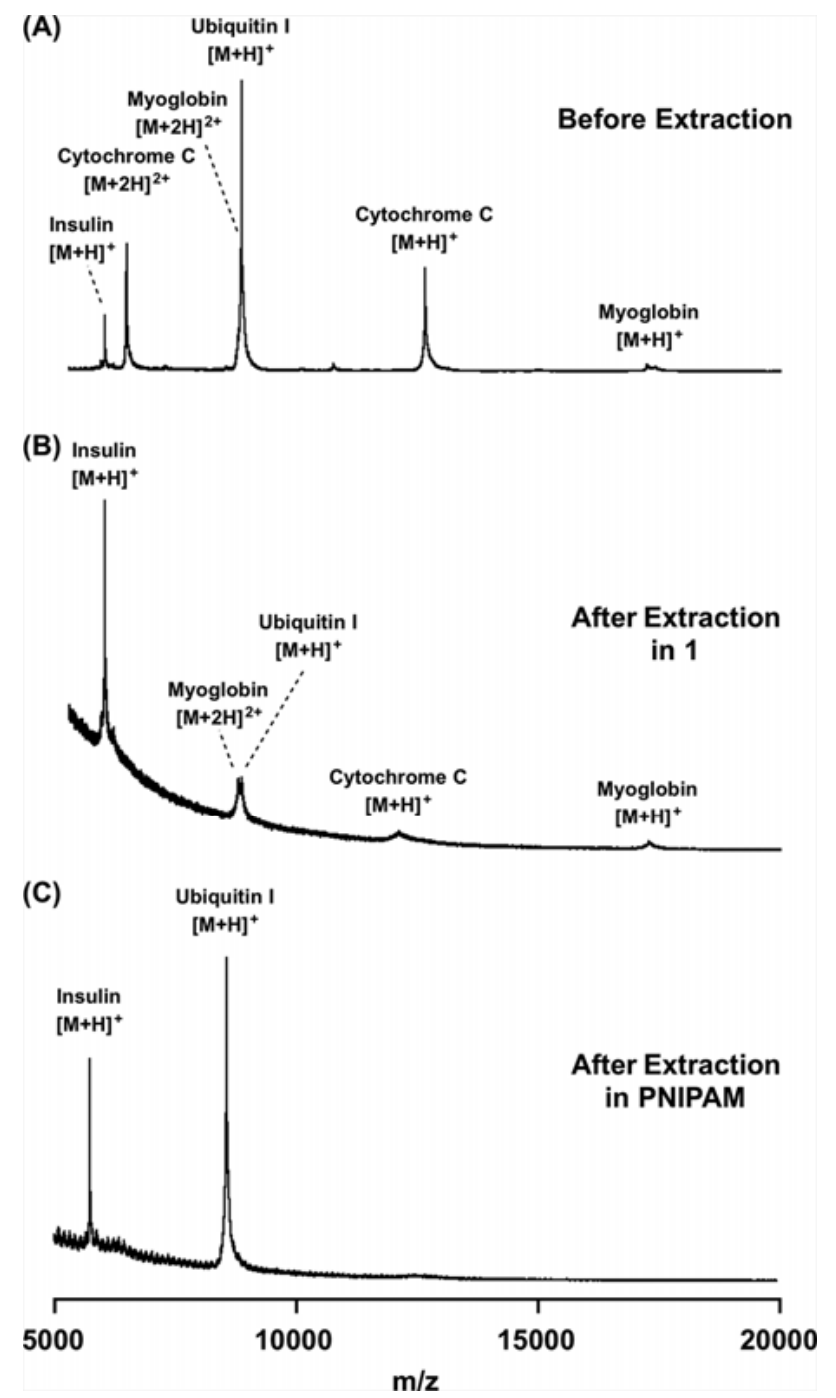

Fig. 2 Matrix-assisted laser desorption/ionization time-of-flight mass spectra of protein calibration standard I (A) before extraction, (B) after extraction with 1 and (C) after extraction with $\operatorname{poly}(N$-isopropylacrylamide) (PNIPAM) measured in a linear positive mode. The observed signals were assigned to the contained proteins (insulin, ubiquitin I, cytochrome $\mathrm{C}$, and myoglobin). Matrix: $\alpha$-cyano-4-hydroxycinnamic acid.

at $12000 \mathrm{rpm}$ at $40{ }^{\circ} \mathrm{C}$ to prompt macroscopic two-phase separation between the aqueous and 1 or PNIPAM phases. The phase of $\mathbf{1}$ or PNIPAM was isolated with expedition, and an aliquot of $10 \mu \mathrm{L}$ was diluted with $1.5 \mathrm{~mL} \mathrm{CH}_{2} \mathrm{Cl}_{2}$ and $0.5 \mathrm{~mL} \mathrm{MeOH}$ in a glass test tube. To the resulting mixture 
(3.0 $\mu \mathrm{L})$ was added CHCA $(6.0 \mu \mathrm{L}$ of the saturated solution in a mixture of acetonitrile and $0.2 \%$ TFA aq. $(1: 1 \mathrm{v} / \mathrm{v})$ and $10 \%$ TFA aq. $(3 \mu \mathrm{L}))$. An aliquot of $1.0 \mu \mathrm{L}$ of the resulting mixture was put on the target plate (MTP 384 Target Plate Ground Steel T $\mathrm{F}$, Bruker Daltonics), and air-dried at $20^{\circ} \mathrm{C}$.

For the study on cytochrome $\mathrm{C}$ extraction, cytochrome C $(0.156 \mathrm{mmol})$ was dissolved in $1.0-\mathrm{mL}$ water, and an aliquot of $200 \mu \mathrm{L}$ was transferred into a $1.5-\mathrm{mL}$ Eppendorf centrifuge tube. The subsequent process was carried out in the same manner described above.

\section{Results and Discussion}

$\mathbf{1}$ is an oligoethylene-glycol-based amphiphile that shows temperature-responsive phase separation in aqueous media. The lower critical solution temperature (LCST) of $\mathbf{1}$ is $26.4{ }^{\circ} \mathrm{C}$, as reported previously. ${ }^{6} \quad$ It is suggested that the phase separation of $\mathbf{1}$ in aqueous media is prompted by the gauche-to-anti conformational change at the carbon-carbon bond of the ethylene oxide units to increase the hydrophobicity. PNIPAM is a temperature-responsive polymer that also shows phase separation in aqueous media above $32{ }^{\circ} \mathrm{C}$, which is due to a coil-to-globule conformational change of the polymer chain. ${ }^{1}$ We performed protein extraction with these temperatureresponsive compounds.

A mixture of four proteins (protein calibration standard I purchased from Bruker Daltonics: insulin, ubiquitin $\mathrm{I}$, cytochrome $\mathrm{C}$, and myoglobin) was dissolved in water, which was then extracted with 1 or PNIPAM by heating to $40{ }^{\circ} \mathrm{C}$ to prompt phase separation. The extract was analyzed by MALDI-TOF MS. As shown in Fig. 2, 1 extracts all of the four proteins, showing a preference to extract a wide variety of proteins. In contrast, it was displayed that PNIPAM extracts insulin and ubiquitin I more preferentially than cytochrome C and myoglobin.

The extraction efficiency of $\mathbf{1}$ was investigated for a photo-active protein, cytochrome C. Absorption spectrum of cytochrome $\mathrm{C}$ extracted in

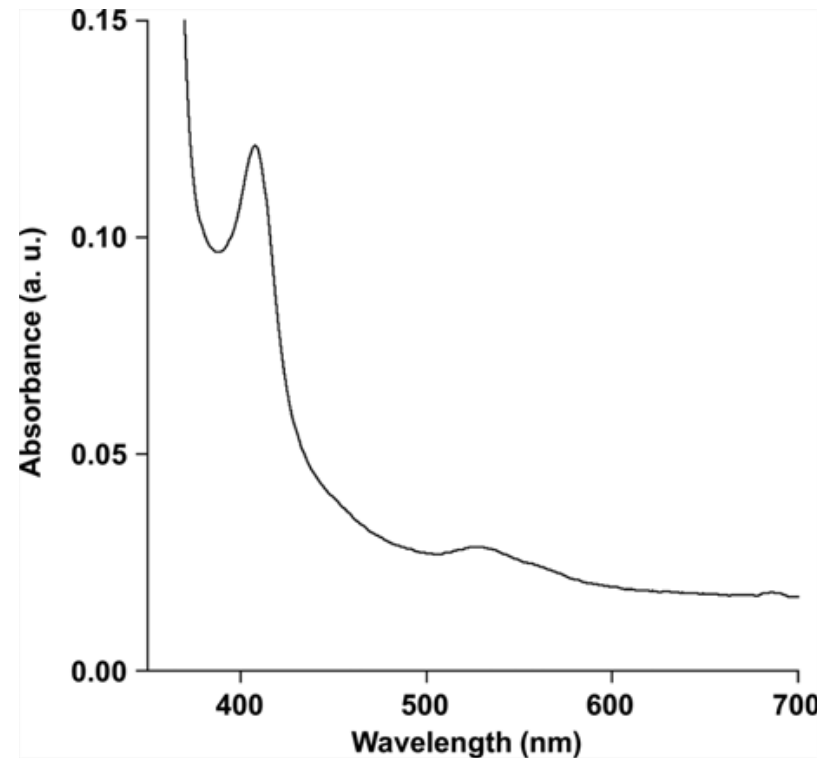

Fig. 3 Absorption spectrum of cytochrome $\mathrm{C}$ in $\mathbf{1}$ at $20{ }^{\circ} \mathrm{C}$. Optical path length: $1.0 \mathrm{~mm}$.

This result suggests that these temperature- responsive substances possess different interaction manners with the proteins at high temperatures.

1 was displayed in Fig. 3, where Soret and Q bands were observed at 408 and $527 \mathrm{~nm}$, respectively. On the basis of the absorption intensity at the Soret band, the extraction efficiency of $\mathbf{1}$ from the aqueous phase was evaluated to be $2 \%$.

\section{Conclusion}

A comparison between temperature-responsive substances 1 and PNIPAM on protein extraction revealed that an oligoethylene glycol based small amphiphile 1 and a polymeric material PNIPAM shows different extraction preferences, where $\mathbf{1}$ has a wider extraction capability including photo-active proteins such as cytochrome $\mathrm{C}$ compared to PNIPAM.

\section{References}

1. S. Fujishige, K. Kubota and I. Ando, J. Phys. Chem. 93 (1989), 3311.

2. H. Kanazawa, T. Sunamoto and Y. Matsushima, Anal. Chem. 72 (2000), 5961; H. Kanazawa, T. Sunamoto, E. Ayano, Y. Matsushima, A. Kikuchi and T. Okano, Anal. Sci. 18 (2002), 
45; T. Niidome, M. Yamagata, Y. Okamoto, Y. Akiyama, H. Takahashi, T. Kawano, Y. Katayama and Y. Niidome, J. Controlled Release 114 (2006), 343; T. Kawano, Y. Niidome, T. Mori, Y. Katayama and T. Niidome, Bioconjugate Chem. 20 (2009), 209.

3. C. Matsubara, N. Kikuchi, I. Denpouya and K. Takamura, Chem. Lett. (1993), 849; C. Matsubara, S. Izumi, K. Takamura, H. Yoshioka and Y. Mori, Analyst 118 (1993), 553; T. Saitoh, T. Ohyama, K. Takamura, T. Sakurai, T. Kaise and C. Matsubara, Anal. Sci. 13 (1997), 1; K. Fujinaga, Y. Yamoto, Y. Seike and M. Okumura, Anal. Sci. 13 (1997), 141; T. Saitoh, K. Asano and M. Hiraide, J. Hazard.
Mater. 185 (2011), 1369.

4. T. Saitoh, Y. Yoshida, T. Matsudo, S. Fujiwara, A. Dobashi, K. Iwaki, Y. Suzuki and C. Matsubara, Anal. Chem. 71 (1999), 4506.

5. M. Hiraide and A. Morishima, Anal. Sci. 13 (1997), 829; T. Saitoh, T. Ohyama, T. Sakurai, T. Kaise, K. Takamura, Y. Suzuki and C. Matsubara, Talanta 46 (1998), 541; T. Saitoh, M. Haga, T. Sakurai, T. Kaise and C. Matsubara, Anal. Sci. 14 (1998), 929; T. Saitoh, S. Ohkubo and C. Matsubara, Chem. Lett. (1999), 151.

6. S. Kawasaki, T. Muraoka, H. Obara, T. Ishii, T. Hamada and K. Kinbara, Chem. Asian J. 9 (2014) 2778. 